\title{
DISRUPCIÓN TECNOLÓGICA Y ORDEN GLOBAL
}

\author{
Felix Arteaga ${ }^{1}$ \\ Instituto Universitario "General Gutiérrez Mellado"/ Real Instituto Elcano
}

\begin{abstract}
Resumen:
Mientras desaparece el orden liberal de postguerra y no acaba de crecer el nuevo orden global, la geopolítica regresa y las grandes potencias utilizan sus instrumentos económicos y tecnológicos para reforzar su capacidad de influencia global. El regreso a la geopolítica se nutre del acelerado proceso de cambio tecnológico en curso y de la carrera de las grandes potencias por controlar los nuevos desarrollos tecnológicos. Este artículo analiza el impacto de la disrupción tecnológica sobre la dinámica de competición geopolítica entre Estados Unidos, China y la Unión Europea
\end{abstract}

Palabras Clave: Geopolítica, digitalización, disrupción tecnología, regionalización, autonomía estratégica

Títle in English: Technological Disruption and Global Order

\begin{abstract}
:
While the liberal post-war order disappears and the new global order hasn't matured, geopolitics returns and the great powers use their economic and technological instruments to strengthen their global influence. The return to geopolitics is nourished by the ongoing process of technological change and the race of the great powers to control new technological developments. This chapter analyzes the impact of the technological disruption on the dynamics of geopolitical competition among the United States, China and the European Union.
\end{abstract}

Key Words: Geopolitics, digitalization, technological disruption, regionalization, strategic autonomy,

Copyright $\odot$ UNISCI, 2019.

Las opiniones expresadas en estos artículos son propias de sus autores, y no reflejan necesariamente la opinión de UNISCI. The views expressed in these articles are those of the authors, and do not necessarily reflect the views of UNISCI.

\footnotetext{
${ }^{1}$ Félix Arteaga es Investigador principal de Seguridad y Defensa, Real Instituto Elcano. Profesor del Instituto Universitario" General Gutiérrez Mellado"

E-mail: <farteaga@ @rielcano.org>

DOI: http://dx.doi.org/10.31439/UNISCI-60
} 


\section{Introducción}

En el pasado, la competencia económica entre las grandes potencias se ha visto moderada por la existencia del orden económico liberal nacido tras la Segunda Guerra Mundial. Sin embargo, recientemente China, Estados Unidos o Rusia cuestionan el multilateralismo, las reglas e instituciones que han regido las relaciones internacionales desde entonces y actúan de forma selectiva -cuando no unilateral- respecto a los grandes asuntos como la economía de mercado, el comercio, el medio ambiente o los derechos humanos que formaban parte del orden liberal resultante de la postguerra ${ }^{2}$.

Las grandes potencias se aprovechan de su mayor capacidad de control de los instrumentos económicos de la economía global, convirtiendo la dependencia de otros en un arma ('weaponized interdependence' según Farrell and Newman ${ }^{3}$ ). El nuevo orden económico ve cómo las grandes potencias coaccionan a las demás sin que éstas puedan recurrir a instrumentos multilaterales o a medidas nacionales de retorsión eficaces. El control de las instituciones económicas, de los sistemas de pago o de los sistemas operativos proporciona a algunos grandes Estados o empresas una capacidad de coacción extraterritorial que se comienza a emplear en la nueva geopolítica. Así, por ejemplo, Estados Unidos ha amenazado con sanciones extraterritoriales a los países y empresas que negocien con Irán, incluso cuando las sanciones estadounidenses no son legales como la de su retirada del Plan Integral de Acción Conjunto (Joint Comprehensive Plan of Action, JCPOA), un acuerdo para prevenir la proliferación nuclear en Irán ratificado por el Consejo de Seguridad de Naciones Unidas. ${ }^{4}$

La 'geopolitización' de los sistemas globales de intercambio conlleva el riesgo de su fragmentación (regionalización) con la consiguiente contracción económica o el de desembocar en una escalada de confrontación económica en que los implicados difícilmente pueden prever y controlar sus consecuencias. ${ }^{5}$ La fragmentación es una aspiración de los sectores más nacionalistas de ambos países que buscan fomentar la desglobalización, desvincular sus economías, separar sus cadenas de suministro y afrontar un futuro bipolar por separado, para lo cual no dudan en obligar a propios y extraños a tomar partido. ${ }^{6}$ En ausencia de un orden global donde las acciones hostiles conlleven penalizaciones disuasorias, se corre el riesgo de que la certeza de la impunidad anime a tomar decisiones poco calculadas o a adoptar respuestas desproporcionadas.

La geopolitización del orden global se manifiesta actualmente en diferentes frentes como el del comercio, donde el proteccionismo de la Administración del presidente Trump plantea guerras comerciales a sus rivales y aliados por igual ${ }^{7} \mathrm{o}$ en el emergente de la guerra de divisas. ${ }^{8}$ Sin embargo, este artículo se va a centrar en la dimensión tecnológica de la geopolítica porque es una variable autónoma que condiciona la dinámica de la (des)gobernanza económica global. Para ello, se va a analizar el comportamiento de tres potencias: Estados Unidos, China y Rusia, seleccionadas porque representan tres modelos de competir diferenciados. El primero, liderado por las grandes empresas y apoyado por las Administraciones que consideran su

\footnotetext{
${ }^{2}$ Mearsheimer, John J: "Bound to Fail: The Rise and Fall of the Liberal International Order", International Security, vol. 43, nº 4 (primavera 2019), pp. 7-50.

${ }^{3}$ Farrel, Henry y Newman, Abraham L.: "Weaponized Interdependence: How Global Economic Networks Shape State Coercion", International Security, vol. 44, no 1 (verano 2019), pp. 42-79.

${ }^{4}$ Joint Call of European Think-Tanks: "The US and the Iran Nuclear Deal: rejoining is wiser than destroying, Elcano Royal Institute, 8 de junio de 2019, en http://realinstitutoelcano.org.

${ }^{5}$ European Political Strategic Centre, "Rethinking Strategic Autonomy in the Digital Age", EPSC Strategic Notes, no 30 (julio 2019), p. 1, en https://ec.europa.eu/epsc.

${ }^{6}$ Editorial: "Business cannot ignore the US-China Cold War", Financial Times, 10 de junio de 2019.

${ }^{7}$ Schneider-Petsinger, Marianne: "US-EU Trade Relations in the Trump Era Which Way Forward?", Chatham House US and the Americas Programme, March 20 (mayo 2019), en http://chathamhouse.org

${ }^{8}$ Plender, John: "The threat of a US-China currency war", Financial Times, 6 de Agosto de 2019.
} 
incremento de poder beneficia a la seguridad nacional. El modelo chino está liderado por el Gobierno e instrumentaliza las empresas nacionales para incrementar su poder global. Finalmente, y aunque no encaja en el concepto geopolítica de potencia, ni está dotado del poder para competir de los anteriores, se ha escogido la Unión Europea porque desarrolla un modelo reactivo de competición para no retroceder en la jerarquía global de competitividad y bienestar. Las demás potencias, como la Federación Rusa, Japón, Francia o India, entre otras, se podrían incluir en alguna de las categorías anteriores, pero su casuística desbordaría el propósito de este estudio, por lo que se ha descartado su inclusión.

\section{La dinámica tecnológica en el orden global}

La nueva carrera tecnológica, a caballo del tecnonacionalismo y del tecnoglobalismo ${ }^{9}$, se libra en un ambiente interconectado en el que todos los instrumentos económicos, las instituciones y las infraestructuras pueden utilizarse como armas en la guerra económica gracias a las tecnologías y redes de comunicaciones. ${ }^{10}$

Las sociedades industriales y postindustriales han convivido en el orden liberal que ahora abandonan adentrándose, ya como sociedades de la información, en un nuevo entorno digital. La digitalización, y las tecnologías disruptivas asociadas a ellas, están cambiando las formas en las que los actores públicos y privados se relacionan, consumen o producen, en lo que se conoce como la Cuarta Revolución Industrial. ${ }^{11}$ La digitalización y las nuevas tecnologías (habilitadores digitales) provocan un cambio cualitativo en la competencia económica porque permiten a quienes innovan antes las tecnologías disponibles o a quienes acceden primero a las nuevas tecnologías desplazar a los competidores en la economía digital. ${ }^{12}$ La preminencia tecnológica favorece la productividad de las compañías y sus tendencias oligopólicas, concentrando la innovación, el desarrollo y la explotación en manos de unas pocas compañías globales. Las compañías y países que no pueden seguir el ritmo de inversión e innovación de las anteriores se ven relegados a nichos de mercado secundarios o condenados a situaciones de dependencia tecnológica, con la consiguiente pérdida de prosperidad y estabilidad, por lo que los gobiernos se ven obligados a considerar la disrupción tecnológica como un problema de seguridad nacional. ${ }^{13}$

No todas las nuevas tecnologías están maduras ni se conoce cuando desarrollarán el potencial transformador que se les atribuye, pero su credibilidad viene avalada por el alto ritmo de aceleración tecnológica que se genera en las economías digitales. La celeridad es importante porque quien accede primero a una tecnología puede convertirla antes en productos y explotar su ventaja con el consiguiente riesgo de desplazar o excluir del mercado a sus competidores (the winner takes it all). Pero al mismo tiempo, se acelera el riesgo de obsolescencia de las tecnologías y los productos asociados a ella, por lo que los países y empresas con menor capacidad de innovación pierden competitividad en la economía digital. La combinación de los factores anteriores aumenta las posibilidades de convertirse en ganadores o perdedores bajo la

\footnotetext{
${ }^{9}$ En este artículo se aplica un enfoque tecnonacionalista que atribuye el mérito de la innovación tecnológica a las naciones, un enfoque controvertido porque no todas las innovaciones se deben a los Estados en concreto, como tampoco se deben al proceso general de globalización. Una cautela para tener en cuenta según Edgerton, Davies: "The Contradictions of Techno-Nationalism and Techno-Globalism. A Historical Perspective", New Global Studies, vol. 1, no 1 (enero 2007), pp. 34-39.

${ }^{10}$ Leonard, Mark (ed.): “Conectivity Wars”, European Council of Foreign Relations (enero 2016), pp. 13-21, en https://ecfr.eu/.

${ }^{11}$ Schwab, Klaus (2017): The Fourth Industrial Revolution, Nueva York, Crown Business.

${ }^{12}$ Entre otras, la UE ha identificado como tecnologías críticas (key enabling technologíes, KET) para su futuro industrial las de materiales avanzados, nanotecnología, micro y nanoelectrónica, biotecnología y fotónica.

${ }^{13}$ Arteaga, Félix: "La cuarta revolución industrial (4RI): un enfoque de seguridad nacional", Documento de Trabajo del Real Instituto Elcano, no 12 (junio 2018), en http://realinstitutoelcano.org.
} 
nueva revolución, lo que explica que los actores internacionales se preocupen por las posibles repercusiones y adopten medidas para paliarlas.

El cambio tecnológico de las últimas décadas y la competencia por el dominio de las nuevas tecnologías de la información está cambiando las relaciones de poder, y no sólo entre las grandes potencias a la que se refiere este artículo, sino a las relaciones entre estados, empresas e individuos. El cambio obedece a que esas nuevas tecnologías proporcionan a quienes acceden a ellas unos instrumentos de influencia disruptivos que permiten cuestionar la jerarquía tradicional entre los anteriores y han puesto en marcha un proceso por el que "el poder se transfiere y redistribuye en todo el planeta con consecuencias difíciles de prever". ${ }^{14} \mathrm{~A}$ medida que se hacen visibles los efectos de la trasferencia y la redistribución, los grandes actores internacionales van tomando consciencia de que el proceso genera perdedores y ganadores y comienzan a tomar posiciones respecto al nuevo orden geopolítico global.

Como resultado, se asiste a una carrera tecnológica entre China y Estados Unidos, entre Asia-Pacífico y Occidente y entre las grandes empresas tecnológicas de la economía digital y las empresas multinacionales de la revolución industrial. La carrera conlleva el diseño e implementación de estrategias y políticas de desarrollo tecnológico, por ejemplo, las que tienen que ver con la investigación, desarrollo e innovación de la inteligencia artificial; pero también con la adopción de medidas proteccionistas o de competencia desleal para denegar o retrasar el acceso de los rivales a la superioridad tecnológica, como revela el caso de Huawei y las plataformas digitales de quinta generación $(5 \mathrm{G})$ que se explica más abajo.

La disciplina de las Relaciones Internacionales, tras un esfuerzo intenso para analizar los aspectos estratégicos durante la Guerra Fría, se ha mantenido al margen de la evolución científica y tecnológica reciente. ${ }^{15}$ La mayor parte de la producción académica sobre ciencia y tecnología corresponde a otras disciplinas científicas aunque la literatura internacionalista ha comenzado a tomar en cuenta sus implicaciones para la comunidad internacional, aunque no lo ha hecho todavía a la altura de la transformación que se avecina, por lo que deberá realizar un esfuerzo importante si quiere comprender el significado y dinámica de la denominada "nueva Guerra Fría". ${ }^{16}$ Tal vez, la razón por la que la disciplina de las Relaciones Internacionales no se ocupado suficientemente de la disrupción tecnológica obedezca a que el impacto de las tecnologías innovadoras sigue siendo más 'potencial' que tangible y que la disciplina se ocupa más de escenarios presentes que de futuro. ${ }^{17}$ En cualquier caso, la bibliografía internacionalista comienza a contar con las primeras valoraciones de la dinámica tecnológica en las relaciones internacionales. Entre las implicaciones preliminares se encuentran la consideración de la ciencia y la tecnología como un elemento disruptivo (game changer) del sistema internacional, que desdibuja los postulados teóricos tradicionales de la disciplina, que crea nuevos riesgos sistémicos y que exige un nuevo nivel macro de análisis de las relaciones internacionales. ${ }^{18}$ Los estudios de seguridad también comienzan a prestar atención al impacto de las nuevas tecnologías en la forma de hacer la guerra o controlar el armamento, pero todavía no se ha

\footnotetext{
14 "The Impact of the ICT Revolution on International Relations", en Strategic Survey 2018: The Annual Assessment of Geopolitics, Londres, IISS, 2018, p. 34.

${ }^{15}$ Mayer, Maximilian: “The Global Politics of Science and Technology: An Introduction”, en Mayer Maximilian; Carpes, Mariana y Knoblich, Ruth (eds.) (2014): The Global Politics of Science and Technology, vol.1, Mayer, Springer, pp. 2-3.

${ }^{16}$ Yuan, Li: "As Huawei Loses Google, the U.S.-China Tech Cold War Gets Its Iron Curtain”, The New York Times, 20 de mayo de 2019.

${ }^{17}$ Danilin, Ivan V.: "Emerging Technologies And Their Impact On International Relations and Global Security", Hoover Institution Fall Series, n $^{\circ} 118$ (octubre 2018), en https://www.hoover.org/publications.

${ }^{18}$ Weiss, Charles: "How Do Science and Technology affect International Affairs", Minerva: A Review of Science, Learning and Policy, vol. 53, $\mathrm{n}^{\circ} 4$ (diciembre 2015), pp. 411-430.
} 
analizado suficientemente el impacto 'relacional' de las tecnologías en las políticas internacionales de exterior y seguridad. ${ }^{19}$

La autonomía o dependencia de las nuevas tecnologías habilitadoras de la digitalización alteran la jerarquía y los factores tradicionales de poder, proporcionando a sus poseedores ventajas decisivas en los ámbitos económico, social e industrial. Por ejemplo, se estima que la inteligencia artificial puede aportar 16 trillones de dólares a la economía global en 2030, aproximadamente un $10 \%$ del producto mundial bruto, cuya distribución entre países y empresas dependen del nicho de la inteligencia artificial que controlen, ya que no todos añaden el mismo valor. ${ }^{20}$ De ahí que las grandes potencias, como las que se mencionan en este artículo, han entrado en un proceso de competencia tecnológica, bien sea en modo ofensivo como China y Estados Unidos, utilizando agresivamente sus instrumentos económicos para conseguir sus fines geopolíticos o en modo defensivo como la UE, tratando de preservar la mayor autonomía posible.

Mientras tanto, se desarrollan tecnologías que tienden a acentuar el poder de las grandes potencias y empresas, Entre aquellas con potencial disruptivo se pueden mencionar los equipos móviles conectados a Internet, la automatización del conocimiento (inteligencia artificial), el Internet de las cosas (IoT), las tecnologías en la nube (cloud), la robótica avanzada, los vehículos autónomos, la genómica, el almacenamiento de energía (baterías), la fabricación aditiva (impresoras 3D) o los materiales avanzados o las energías renovables. ${ }^{21}$ La disrupción de todas estas tecnologías se alimenta de los progresos alcanzados en los sistemas de información y comunicaciones (TIC) o en las nuevas capacidades de supercomputación y de computación cuántica que amplifican y aceleran la interacción entre todas las anteriores.

Las sociedades avanzadas tienen que correr riesgos si quieren progresar y la aceleración tecnológica acelera el riesgo global de las sociedades que anunciara Ulrich Beck. ${ }^{22}$ Sociedades avanzadas como las de Estados Unidos, China y la Unión Europea se asoman al riesgo asociado a la implantación de tecnologías y servicios cuyas contraindicaciones no se conocen todavía, aunque algunas de ellas ya se utilizan como instrumentos de agresión. Los ciberataques, la desinformación o la guerra híbrida forman parte de los inventarios de agresión y competencia desleal en la carrera tecnológica y se comienzan a combinar con el análisis de datos, la inteligencia artificial o la automatización de los sistemas de armas. ${ }^{23}$ La resiliencia, es decir, la capacidad de reponerse tras recibir este tipo de ataques ha reemplazado a la vieja idea de la seguridad que pretendía evitarlos.

Las tecnologías aplicadas al ciberespacio permiten a las grandes potencias -y a las pequeñas como Corea del Norte- que disponen de servicios adecuados desarrollar y aplicar instrumentos cibernéticos para recabar información y datos de sus competidores, pero también para espiar y hacerse con activos relacionados con la propiedad intelectual o depósitos bancarios o introducir elementos maliciosos (exploits) en las infraestructuras críticas que faciliten información y, en su caso, el sabotaje de las mismas como ocurrió con las centrifugadoras iraníes mediante el virus Stuxnet en 2010. Desde entonces, los ciberataques no paran de crecer y de poner en riesgo la digitalización de la economía. A su autoría contribuyen

\footnotetext{
${ }^{19}$ Bueguer, Christian: "Security as practice" y Cavelty, Myriam Dunn; Fischer, Sophie-Charlotte y Balzaq, Thierry: "Killer Robots and Preventive Arms Control" en Myriam Dunn Cavelty (ed.) (2018): Routledge Handbook of Security Studies, Taylor and Francis, pp. 131 y 457-459, respectivamente.

${ }^{20}$ Stanton, Charlotte et al.: "What the Machine Learning Value Chain Means for Geopolitics", Carnegie Endowement, agosto 2019, en https://carnegieendowment.org.

21 "Disruptive technologies: Advances that will transform life, business, and the global economy", McKinsey Global Institute (mayo 2013), pp. 4-5.

${ }^{22}$ Beck, Ulrich (2006): La sociedad del riesgo global, Madrid, Siglo XXI.

${ }^{23}$ ESPC, op. cit., p. 5.
} 
actores gubernamentales o paragubernamentales, las denominadas amenazas avanzadas persistentes que desarrollan instrumentos cibernéticos para manipular, denegar, perturbar, degradar o destruir sistemas de información e infraestructuras críticas de los rivales. Más allá de las capacidades de defense y resiliencia cibernética que desarrollan los Estados y las empresas, los estados desarrollan capacidades ofensivas (defensa activa) para disuadir a los posibles agresores. Grandes potencias Estados Unidos, China y Rusia, pero también potencias menores como el Reino Unido, Australia, Holanda o Dinamarca forman parte de la treintena de países que en 2016 ya desarrollaban las nuevas capacidades ofensivas. ${ }^{24}$

La disrupción tecnológica afecta al equilibrio de poder porque las ventajas comparativas adquiridas con la investigación y desarrollo de armamento por las grandes potencias y sus industrias nacionales se pierden cada vez más deprisa. Potencias de menor tamaño e, incluso actores no estatales, acceden ahora a tecnologías que fueron una vez de monopolio occidental como las municiones de precisión guiadas o los vehículos aéreos no tripulados, y exploran otras tecnologías de naturaleza dual que les permiten contrarrestar la superioridad tradicional de las grandes complejos militares-industriales. Cada vez son más los actores que acceden a tecnologías que dificultan o impiden la proyección de fuerzas (Anti-Access, Area Denial, $\mathrm{A} 2 / \mathrm{AD}){ }^{25}$

La carrera tecnológica afecta también a la forma de hacer la guerra, tanto en los dominios tradicionales de tierra, mar y aire, como en los nuevos del ciberespacio y del espacio. La inteligencia artificial acelera el proceso de decisiones de los mandos y de los sistemas de armas, la hipervelocidad favorece a los sistemas ofensivos, el aprendizaje automático (deep learning) favorece la aparición de sistemas autónomos, y los combatientes individuales y las plataformas de combate acceden a cantidades ingentes de datos procesados mediante la inteligencia artificial y transmitidos mediante las nuevas tecnologías de comunicación. Las tecnologías mejoran la capacidad de mando, control, comunicaciones, computación, inteligencia, vigilancia y reconocimiento (C4ISR), el conocimiento situacional del entorno del combate, la autonomía y resiliencia de las unidades de combate. Permiten reducir la presencia de los combatientes sobre el terreno, aumentar la distancia desde la que se combate y actuar en una zona gris entre la paz y la guerra (guerra híbrida). La disrupción en el campo militar ya está en marcha y mientras se discuten en Naciones Unidas los aspectos éticos y jurídicos de los nuevos sistemas de armas, países como Estados Unidos o China están investigando y desarrollando nuevos sistemas de armas alterando los equilibrios tradicionales de fuerzas convencionales y nucleares. $^{26}$

\section{Los Estados Unidos}

Estados Unidos invierte una parte importante de su producto interior bruto en investigación y desarrollo: el 3\% en 2015 con una cifra aproximada de 500 billones de dólares, del que la parte principal $(60 \%)$ corresponde al sector privado miembros de las nuevas tecnologías. El resto corresponde al sector público que lo dedica principalmente a la investigación básica, pero su porcentaje se va reduciendo progresivamente, lo que permite a sus competidores recortar la ventaja tecnológica. ${ }^{27}$ La tendencia afecta a la competitividad general de la economía

\footnotetext{
${ }^{24}$ Uren, Tom; Hogeveen, Bart y Hangus, Ferson: “Defining offensive cyber capabilities”, Australian Strategic Policy Institute, 4 de julio de 2018, en https://www.aspi.org.au.

${ }^{25}$ Freier, Nathan: "The Emerging Anti-Access/Area-Denial Challenge”, CSIS Critical Questions, 17 de mayo de 2012, en https://www.csis.org

${ }^{26}$ Conn, Ariel: "The United Nations and the Future of Warfare", Bulletin of the Atomic Scientist, 9 de mayo de 2019, en https://thebulletin.org.

${ }^{27}$ Eagler, John y Pritzker, Penny: "The Work Ahead: Machines, Skills, and U.S. Leadership in the Twenty-First Century”, Council on Foreign Relations Independent Task Force, Report, nº 76, (abril 2018), pp. 16-17, en https://cfr.org.
} 
estadounidense, salvo la de las grandes compañías tecnológicas y al conjunto de la seguridad nacional, una tendencia que sólo ha cambiado cuando las últimas Administraciones de Estados Unidas han tomado consciencia de los riesgos de las tecnologías disruptivas para su liderazgo económico y militar. Así, por ejemplo, el sector privado lideró la investigación en inteligencia artificial hasta 2016, invirtiendo entre 20 y 30.000 millones de dólares frente a los 1.100 millones de dólares del sector público. La situación cambió a partir de 2018, cuando la Administración tomó medidas para impulsar la investigación y el desarrollo de la inteligencia artificial como un objetivo prioritario para la prosperidad y seguridad nacional, tal y como se recoge en las Prioridades Presupuestarias para $2020^{28}$, en la Estrategia de Seguridad Nacional ${ }^{29} \mathrm{o}$ en la Evaluación de la Base Industrial del mismo año ${ }^{30}$.

El peso del sector privado se traduce en un enfoque de innovación orientado a la producción y el mercado. Estados Unidos son la gran potencia digital del momento, cuenta con cinco de las más grandes empresas tecnológicas del mundo (Alphabet, Amazon, Apple, Facebook y Microsoft) inmersas en un proceso de expansión global y de compra de competidores. Controla las instituciones más importantes de gobernanza de Internet: el consorcio de la red mundial (World Web Consortium), el estandar linguístico (Unicode Consortium) y la asignación de direcciones y dominios (Internet Corporation for Assigned Names and Numbers, ICANN).

La defensa nacional ha sido uno de los sectores en los que las tecnologías disruptivas han generado mayor preocupación. Ya en noviembre de 2014, el Departamento de Defensa lanzó su Defence Innovation Initiative, conocida como la 'Third Offset Strategy', en la que se identificaban tecnologías críticas para el combate del futuro como la inteligencia artificial, los metadatos y la nube. ${ }^{31}$ La Agencia de Investigación de la Defensa (Defense Advanced Research Projects Agency, DARPA) centró sus investigaciones en tecnologías disruptivas críticas como las armas hipersónicas o la energía dirigida ${ }^{32}$. En la Estrategia de Defensa Nacional de 2018 se reconoce que las tecnologías como la inteligencia artificial, la computación avanzada, la robótica, la energía dirigida o los metadatos están transformando la forma de hacer la guerra. ${ }^{33}$

Estados Unidos se ha aprovechado de su capacidad digital para desarrollar instrumentos opacos de control de la información que atraviesa el ciberespacio. Su Agencia Nacional de Seguridad (National Security Agency) y su Mando de Ciberdefensa (US Cyber Command) se han visto implicados en una larga cadena de escándalos que van desde escuchas a sus aliados, al acceso indebido a datos privados o a la elaboración de virus informáticos que ha generado la desconfianza sobre su fiabilidad como controlador de Internet. ${ }^{34}$ A ello habría que añadir los excesos provocados por algunas de sus grandes compañías tecnológicas como Google, multada por la UE por su posición dominante en el mercado de la publicidad en línea; o por el uso por

\footnotetext{
${ }^{28}$ Memorandum del Ejecutivo M-18-22 de 31 de julio de 2018 sobre "FY 2020 Administration Research and Development Budget Priorities", en https://whitehouse.gov.

29 "National Security Strategy of the United States of America, 18 de diciembre de 2017, en https://whitehouse.gov.

${ }^{30}$ Interagency Task Force in Fullfillment of the Executive Order 13806, "Assessing and Strengthening the Manufacturing and Defense Industrial Base and Supply Chain Resiliency of the United States" Report to President Donald J. Trump, septiembre 2018, en https://media.defense.gov.

31 "The Defence Innovation Initiative", Memorandum de 15 de noviembre de 2014, Secretaria de Defensa de los Estados Unidos, en https://archive.defense.gov.

${ }^{32}$ Calhoun, Paul: “DARPA Emerging Technologies”, Strategic Studies Quarterly (otoño 2016), pp. 91-113.

33 "National Defence Strategy", sumario no clasificado, Departamento de Defensa de los Estados Unidos, 2018, p.3, en https://dod.defense.gov.

${ }^{34}$ La Agencia Nacional de Seguridad (NSA) accedió ilegalmente a las comunicaciones privadas de nueve servidores de Internet incluidos Facebook, Google, Microsoft o Yahoo. Lee, Thimothy B.: "Here's everything we know about PRISM to date", The Washington Post, 12 de junio de 2013.
} 
Cambridge Analytica de Facebook de más de 50 millones de datos de usuarios de Facebook para cambiar su comportamiento electoral.

Pese a las desviaciones mencionadas, en las que ha primado el interés particular del Gobierno y de las grandes empresas tecnológicas frente a cualquier otra consideración ética, Estados Unidos ha venido mantenido la libertad del mercado tecnológico y el acceso a sus centros de formación e investigación hasta el pasado inmediato. Una libertad que había favorecido la capacidad de innovación e investigación de Estados Unidos pero que la geopolitización comienza a recortar. En los últimos años se han levantado barreras a la trasferencia o adquisición de tecnologías críticas y a la libre circulación de investigadores extranjeros. Las restricciones se justifican por razones de seguridad nacional y, en el caso de China, por la colaboración de empresas como Baidu Tencent y Sensetime o de universidades como la de Beihang por su colaboración con las fuerzas armadas y los servicios de inteligencia, aunque la Secretaría de Comercio ha ido ampliando esas restricciones a la relación de las compañías chinas con el Gobierno, y no sólo por motivos comerciales sino por motivos económicos.

En 2018, los Estados Unidos aprobaron la Ley de Reforma del Control de las Exportaciones (Export Control Reform Act, ECRA) y la Ley de Modernización de la Revisión de Riegos de Inversiones Extranjeras (Foreign Investment Risk Review Modernization Act, FIRRMA) que permiten al Departamento de Comercio o al Comité de Inversiones Extranjeras (Committee on Foreign Investment in the United States, CFIUS) controlar el uso, destino y usuario final de las trasferencias de tecnología o de propiedad intelectual, así como las inversiones extranjeras. Con ello tratan de preservar la superioridad tecnológica de los EE.UU., especialmente en las tecnologías que afectan a la seguridad nacional como la fabricación aditiva, la robótica, las conexiones biotecnologías, la inteligencia artificial, el posicionamiento, los microprocesadores, la computación avanzada, la computación cuántica, los interfaces cerebro-ordenador, los sistemas hipersónicos, los materiales avanzados o los sistemas avanzados de vigilancia, entre otras. ${ }^{35}$ En conjunto, las medidas adoptadas están muy lejos de constituir un régimen de control de tecnologías similar al Comité de Coordinación (Coordinating Committee for Multilateral Export Controls, CoCom) creado durante la Guerra Fría para evitar la trasferencia de tecnología militar o dual al bloque del Este, aunque las restricciones de entonces no afectaban también a sus aliados como las de ahora.

\section{China}

La innovación tecnológica es un objetivo estratégico de China, no sólo para afrontar los retos económicos y sociales internos, sino también para asegurar su liderazgo internacional. Para ello sigue el enfoque tecnonacionalista declarado en su Plan para el Desarrollo de la Ciencia y la Tecnología a medio y largo plazo (2006-2020) mediante el cual utilizar todos sus recursos nacionales tanto para fomentar su capacidad de innovación tecnológica como para recortar la de sus competidores. ${ }^{36} \mathrm{El}$ tecnonacionalismo chino favorece la orientación de las políticas tecnológicas mencionadas tanto hacia el interior: modernizando la economía, reduciendo la dependencia y facilitando el control social, como hacia el exterior: aumentando la penetración tecnológica china en el mercado global, especialmente en el de las infraestructuras y servicios digitales asociados a la iniciativa de la Ruta de la Seda.

\footnotetext{
${ }^{35}$ Government Publishing Office, Federal Register, vol. 83, nº 223, p. 58202.

${ }^{36}$ Kennedy, Scott: “The Fact Tec Dragon. Benchmarking China's Innovation Drive", CSIS China Innovation Policy Series (agosto 2017), p.1, en https://www.csis.org.
} 
El modelo chino corresponde al de una economía dirigida en la que el desarrollo tecnológico se beneficia de los recursos públicos ilimitados. El Estado ha puesto en marcha diversas estrategias y planes de acción como el "Made in China 2025" que pretende reducir su dependencia tecnológica del exterior en esa fecha para alcanzar una posición de dominio global en $2049^{37}$, Para lograrlo, no sólo desarrolla su propia capacidad tecnológica, sino que también trata de reducir la de sus posibles rivales recurriendo al proteccionismo ${ }^{38}$, a la compra de activos estratégicos, a la trasferencia forzosa de tecnologías ${ }^{39} \mathrm{o}$ al espionaje. ${ }^{40}$ China no pone en cuestión el orden económico internacional, pero lo interpreta de formas discriminadas o unilaterales que lo ponen en peligro. Además, y por si algún día se fragmentan los servicios globales económicos y de comunicaciones, China está cooptando apoyos nacionales y empresariales para elaborar una red alternativa a los instrumentos globales controlados por sus rivales que le sirva de protección en caso de fragmentación. ${ }^{41}$

El Gobierno aprobó su plan para el desarrollo de la Inteligencia Artificial y va a invertir 150.000 millones de dólares para liderar la inteligencia artificial en $2030^{42}$. La competición por el liderazgo se sustancia imponiendo los estándares tecnológicos chinos, al igual que hicieron en el pasado las grandes compañías occidentales como Google o Facebook. Convertirse en generador de estándares (standard-setter) en lugar de consumidor de ellos se ha convertido en un instrumento para liderar la competición tecnológica, una política que ha permitido a China competir con ventaja en implantación de las redes digitales de nueva generación (5G) como se verá más adelante en relación con el caso de Huawei.

Para ello, las universidades y centros tecnológicos chinos han centrado sus esfuerzos en traducir sus avances en investigación básica en patentes. Como resultado, China sobrepasó en 2011 a Estados Unidos en el número de patentes y en 2013 sobrepasó a la UE en el gasto en I+D. Según datos de la Organización Mundial de la Propiedad Intelectual para 2017, de los 13,7 millones de patentes en vigor, unos 3 millones correspondían a los Estados Unidos, 2,1 a China y 2 a Japón. Sin embargo, de los aproximadamente 3,2 millones de solicitudes de patentes industriales en 2017, el 43,6\% corresponde a China; seguida del 19,2\% de Estados Unidos, el 10,1\% de Japón, el 6,5\% de la República de Corea y el 5,3\% de los países de la UE. ${ }^{43}$

China ha sabido explotar la predisposición de las comunidades académicas occidentales para intercambiar científicos y estudiantes para desarrollar su tecnología militar, enviando desde 2007 más de 2.500 científicos asociados al desarrollo tecnológico del Ejército de

\footnotetext{
${ }^{37}$ Jost Wübbeke et al:: "Made in China 2025. The making of a high-tech superpower and consequences for industrial countries", Mercator Institute for China Studies, MERICS Papers on China, n 2, December 2016, en https://www.merics.org.

${ }^{38}$ Morrison, Wayne M.: "China-U.S. Trade Issues”, Congressional Research Service (6 de julio de 2018), p. 39.

${ }^{39}$ Lind, Jennifer: "The Rise of China and the Future of Transatlantic Relationship", Chatham House Briefing, 29 de julio de 2019, p. 5.

${ }^{40}$ Feng, Emily: "How China acquired mastery of vital microchip technology, Financial Times, 29 de enero de 2019. Czerwonka, Michal: "How Chinese Spies Got the N.S.A.'s Hacking Tools, and Used Them for Attacks", The New York Times, 6 de mayo de 2019. Sobre el impacto del espionaje de tecnología militar, ver Gilli, Andrea y Gilli, Mauro: "Military-Technological Superiority and the Limits of Imitation, Reverse Engineering, and Cyber Espionage", International Security, vol. 43, n 3 (invierno 2018/19), p. 144.

${ }^{41}$ En este sentido, se viene especulando últimamente con la posibilidad de que China articule su propio Internet, tanto por razones de control interno como de autonomía económica. McDonald, Sean y Mina, An Xian: “The WarTorn Web", Foreign Policy, 19 de diciembre de 2018, en https://foreigpolicy.com.

${ }^{42}$ Consejo de Estado Chino: “A Next Generation Artificial Intelligence Development Plan,”, traducción disponible en https://www.newamerica.org.

43 "Datos y cifras de la OMPI sobre PI", Organización Mundial de la Propiedad Industrial, edición en español, 2018, pp. 9-11.
} 
Liberación Popular en la última década. ${ }^{44}$ El programa de la Universidad Nacional de Tecnología de la Defensa trata de acelerar el trasvase de tecnología civil al campo militar (denominada como 'fusión civil-militar' en China) integrando a sus académicos en el seno de equipos occidentales que trabajan en tecnologías de aplicación militar como el procesamiento de señales, física cuántica, criptografía o posicionamiento (una colaboración en la que participan empresas de Estados Unidos como Microsoft Research $\mathrm{Asia}^{45}$ ). En el campo civil, los grandes conglomerados tecnológicos chinos reclutan tecnólogos occidentales duplicando sus ingresos o asumiendo la financiación de sus proyectos tanto en China como en los centros tecnológicos (hubs) que han abierto en países avanzados. ${ }^{46}$

\section{La Unión Europea}

El modelo de competición europeo se caracteriza por carácter reactivo y defensivo. La UE ha entrado en la competición para defenderse de ella, cuando ha tomado consciencia de su declive tecnológico y económico, y a remolque de las iniciativas de los anteriores, porque carece de liderazgo y capacidad para emularlas. A diferencia de Estados Unidos o China, la Unión Europea sigue diferenciando sus intereses económicos de los geopolíticos, por lo que la UE se encuentra en una situación de riesgo y desventaja en la actual carrera tecnológica en la que se han metido las grandes potencias para asegurar su posición de dominio sobre la economía globalizada. La dependencia europea de tecnología china o estadounidense no para de crecer, especialmente respecto a los semiconductores y microprocesadores necesarios para la inteligencia artificial, la supercomputación o la computación cuántica, así como de tecnologías de software y aplicaciones cloud. La reducción de los proveedores aumenta la exposición pública y privada frente a los grandes proveedores en caso de fallo, ataque o retorsión y coloca su información sensible a merced de terceros que la trasladan, utilizan o almacenan.

Debido a ello, algunos autores han recomendado a la Comisión que diseñe una estrategia para reforzar su soberanía económica reforzando la investigación, protegiendo los activos críticos, fomentando la competencia en igual de condiciones, sin necesidad de incurrir en el proteccionismo o desvincularse de la globalización. ${ }^{47}$ En particular, la Comisión Europea critica la falta de reciprocidad en su relación con China, sus barreras proteccionistas, las restricciones para acceder a su mercado y servicios o la trasferencia forzosa de tecnologías, prácticas con las que China pretende conseguir su liderazgo tecnológico a costa, en este caso, de los europeos. ${ }^{48}$

La Unión Europea, como potencia normativa que es, rechaza las actuaciones unilaterales al margen de los foros multilaterales y reivindica la necesidad de encontrar formas de gobernanza para las nuevas tecnologías. Así, en su Estrategia de Política Exterior y de Seguridad de 2016, reivindica la necesidad de articular normas globales en campos como la biotecnología, la inteligencia artificial, la robótica o los sistemas pilotados a distancia. ${ }^{49}$ Por otra parte, la regulación del mercado interior defiende el libre flujo de personas, capitales y

\footnotetext{
${ }^{44}$ Jaske, Alex: "Picking Flowers, Making Honey", Australian Strategic Policy Institute, 30 de octubre de 2018, en https://aspi.org.au.

${ }^{4}$ Yang, Yuan y Murgia, Madhumita: "Microsoft under fire over AI work with chines military university", Financial Times, 11 de abril de 2019.

${ }^{46}$ Entre otras, Alibaba ha abierto centros en Estados Unidos, Singapur o Israel para desarrollar proyectos de inteligencia artificial, computación cuántica y tecnología financiera; y Tencent ha abierto otro sobre inteligencia artificial en Seattle.

${ }^{47}$ Leonard, Mark et al.: "Redefining Europe's economic sovereignty, Bruegel Policy Contribution, $\mathrm{n}^{\circ} 9$ (junio 2019), en http://bruegel.org.

${ }^{48}$ Comunicación conjunta de la Comisión Europea y de la Alta Representante JOIN (2019) 5 final, 12 de marzo, "European Council EU-China. A strategic outlook", en https://ec.europa.eu/commission.

49 “A Global Strategy for the European Union's Foreign And Security Policy. Shared Vision, Common Action: A Stronger Europe”, Consejo Europeo, junio 2018, en https://eeas.europa.eu.
} 
mercancías a o desde países terceros, por lo que la economía europea depende de los mercados globales. Además, los Estados miembros disponen de un amplio grado de autonomía en el ámbito económico, por lo que a la Unión Europea le resulta difícil adoptar medidas proteccionistas o unilaterales que restrinjan el acceso externo a tecnologías, equipos y conocimientos críticos europeos, unas medidas a las que China y Estados Unidos recurren con mayor agilidad en el nuevo ambiente de competencia geopolítica.

No obstante, la geopolitización reinante ha obligado a la Unión Europea a revisar sus planteamientos de principios para proteger a la tecnología europea y aunque no pretende competir contra las grandes potencias, sí que busca, al menos, reducir la dependencia de terceros en aquellas áreas críticas para la soberanía económica y política. Por un lado, la Unión Europea ha tomado consciencia de su creciente desfase tecnológico y adoptado algunas medidas para reducirlo. La Unión Europea preserva todavía un capital tecnológico importante. Dispone de grandes compañías como SAP o Amadeus, ocho de las veinte grandes empresas tecnológicas tienen sede en Europa y compañías europeas como Ericsson y Nokia copaban el $30.4 \%$ del mercado global en 2018 (Huawei el 29\%). ${ }^{50}$ La UE todavía dispone de una capacidad exportadora de alta tecnología (616,2 billones de dólares en 2016), por delante de China que lidera el escalafón de países exportadores de alta tecnología (504,4 billones de dólares), casi quintuplicando a Estados Unidos (110,2 billones), pero podría perder esa capacidad si no actualiza sus niveles tecnológicos para mantener su competitividad. ${ }^{51}$

Para evitarlo, la Unión Europea ha puesto en marcha programas de investigación y desarrollo de tecnologías orientadas a fomentar la competitividad de las empresas e industrias como la Agenda Digital para Europa de 2014, el Mercado Digital Único de 2016 o la Estrategia para la Política Industrial de 2017 que revelan la preocupación europea por el impacto de los cambios tecnológicos sobre la competitividad industrial ${ }^{52}$. En el mismo sentido, la Comisión ha alertado sobre la importancia de la inteligencia artificial para la competitividad y sobre la necesidad de aunar las capacidades y estrategias nacionales ${ }^{53}$. La coordinación de las capacidades y estrategias nacionales que propone la Comisión permitiría, por ejemplo, sacar mayor partido a la inversión privada europea que es inferior a la de sus competidores: unos 2.500-3.300 millones de euros frente a los 6.500-9.800 de Asia o a los 12.200-18.800 de Estados Unidos 54 .

Por otro lado, la Unión Europea se ha visto en la necesidad de proteger sus activos tecnológicos. La percepción comenzó a cambiar cuando los datos mostraron que, por ejemplo, las inversiones chinas habían crecido no sólo de forma cuantitativa en los últimos años, pasando de 1.600 millones de euros en 2010 a 35.000 millones en 2016, sino sobre todo cualitativamente, orientando las inversiones a la compra de empresas tecnológicas que no disponían de mecanismos nacionales de protección. ${ }^{55}$ Para evitar en lo posible la pérdida de masa tecnológica crítica, los responsables de Economía de Alemania, Francia e Italia pidieron a la Comisión que introdujera mecanismos de supervisión similares a los que se estaban introduciendo en algunos Estados miembros y en Estados Unidos.

\footnotetext{
${ }^{50}$ Véase ESPC, op. cit., p.10.

${ }^{51}$ Datos del Banco Mundial, "High-Tech Exports", en https://data.worldbank.org.

${ }^{52}$ Los textos oficiales: "European Industrial Strategy", "Industry 4.0" y "Digital Single Market" se encuentran en https://ec.europa.eu/commission/priorities/digital-single-market_en.

53 "Inteligencia artificial para Europa", Comisión Europea, Comunicación (2018) 237 final de 25 de abril, en https://ec.europa.eu.

54 "The age of artificial intelligence", European Political Strategy Center, EPSC Strategic Notes no 29,27 marzo 2018 , p. 4.

${ }^{55}$ Ortega, Andrés: “Europa se quita el velo chino”, Blog del Real Instituto Elcano (abril 2019), en https://realinstitutoelcano.org.
} 
La Comisión Europea presentó en septiembre de 2017 su primera propuesta para supervisar las inversiones directas en la UE que condujo, en febrero de 2019, a la aprobación del Reglamento que permite filtrar las inversiones directas extranjeras en tecnologías críticas para la seguridad nacional de los Estados miembros. ${ }^{56}$ Entre ellas, figuran las tecnologías aplicables a las Infraestructuras críticas, la inteligencia artificial, la robótica, los semiconductores, la ciberseguridad, la defensa, el almacenaje de energía, el sector aeroespacial, nanotecnología, biotecnología. Un largo listado sobre el que la Comisión Europea tiene limitadas capacidades de actuación, porque son los Estados miembros los que deben velar por la protección de su seguridad nacional y no existe unanimidad sobre la necesidad ni la forma de protegerla. Así, mientras que la mitad de los Estados miembros disponen de ya algún tipo de control y que algunos países como el Reino Unido, Francia o Alemania han tomado medidas específicas frente a las inversiones chinas, otros como Italia, Portugal, Hungría y Malta se resisten a aplicarlas porque se benefician de ellas ${ }^{57}$. En esas condiciones, el mecanismo de coordinación no puede ser vinculante y la Comisión Europea solo puede recomendar medidas de coordinación entre ellos o tomar la iniciativa cuando las inversiones extrajeras afectan a varios países o ponen en riesgo proyectos europeos como el Galileo, el Horizonte 2020 o el futuro Horizonte Europa. Finalmente, y como se ha mencionado anteriormente, la Comisión Europea ha comenzado a tomar medidas contra las prácticas monopolísticas de las grandes compañías tecnológicas de los Estados Unidos, no sólo para proteger a los consumidores sino para evitar que el abuso de las plataformas deteriore la competencia dentro del mercado único. ${ }^{58}$

Individualmente, el Reino Unido endureció los controles sobre la compra de empresas que afectan a la seguridad nacional en junio de 2018, modificando su legislación empresarial (Enterprise Act de 2002) para aumentar la capacidad de intervención del Gobierno en la venta de empresas dedicadas a tecnologías de doble uso, hardware y computación. Pese a que hasta ahora el número de intervenciones del Gobierno ha sido muy limitada, por ejemplo, para denegar la compra de Northern Aerospace por la compañía Gardner Aerospace participada por China en junio de 2018, aunque se espera que el régimen de intervención por razones de seguridad nacional se vaya ampliando en los próximos años. ${ }^{59}$

Por su parte, los gobiernos franceses han seguido una aproximación similar a la británica y han consensuado una revisión legislativa (Le Plan d'Action pour la Croissance et la Transformation des Entreprises, Ley PACTE) de las normas para hacer más competitivas a las industrias francesas. En ella se amplía el régimen de autorización previa y de sanciones en el caso de ciertas tecnologías críticas para la seguridad nacional como las relacionadas con el espacio, los semiconductores, los drones, la inteligencia artificial, la robótica, la ciberseguridad y el almacenamiento de datos. ${ }^{60}$ De esta forma, los gobiernos franceses podrán ampliar el control que ya ejercen sobre las empresas nacionales en las que poseen un mecanismo de control estratégico (acción de oro), una práctica de los Estados miembros contra la cual la Comisión Europea se ha mostrado siempre beligerante hasta ahora.

\footnotetext{
${ }^{56}$ Reglamento (UE) 2019/452 del Parlamento Europeo y del Consejo, de 19 de marzo de 2019, para el control de las inversiones extranjeras directas en la Unión, en https://eur-lex.europa.eu.

57 "Expanding the Scope of National Security-Focused Foreign Investment Reviews in Europe", julio 2018, Informe de la firma de abogados Skadden, en https://www.skadden.com.

${ }^{58}$ Cremer, Jacques; De Montjoye, Yves-Alexandre y Heike Schweitzer, Heike: "Competition policy for the digital age", Comisión Europea, abril de 2019, en http://ec.europa.eu/competition. Informe analizado por Yoplensky, Rochelle y Murgia, Madhumita: "EU antitrust crackdown on Big Tech Urged", Financial Times, 5 de abril de 2019.

${ }^{59}$ Potter, Alex: "United Kingdom", en Goldman Calvin S.(ed.) (2018): The Foreign Investment Regulation Review, sexta edición, Londres, Law Business Research Ltd, pp. 195-226.

60 "La croissance et la transformation des entreprises", Ley 486 (2019) de 22 de mayo, en https://www.legifrance.gouv.fr.
} 


\section{El caso-estudio de Huawei}

La oposición estadounidense a la presencia de tecnología china en sus infraestructuras digitales de quinta generación (5G) puede servir para validar el estado y manifestaciones de la competencia tecnológica en curso porque presenta alguna de las variables de confrontación geopolítica señaladas: una creciente rivalidad tecnológica, la utilización de instrumentos discriminatorios y la implicación de terceros en la confrontación.

Las redes móviles de quinta generación $(5 \mathrm{G})$ representan un salto cualitativo respecto a las de generaciones anteriores. La velocidad de transmisión y descarga será unas 100 veces mayor y dispondrá de mayor capacidad para facilitar la comunicación entre máquinas y acceder a grandes bases de datos. Las redes $5 \mathrm{G}$ añadirán valor a la economía digital de los países donde se instalen y generarán ingentes beneficios económicos a los propietarios de las infraestructuras $\mathrm{y}$, sobre todo, a los desarrolladores de aplicaciones y servicios que permitan aprovechar el potencial de las nuevas redes. Dado su impacto económico, no es de extrañar que a presencia de compañías y tecnologías chinas en las redes o su interés por participar en ellas haya despertado un nuevo capítulo en la rivalidad geopolítica entre ambas potencias y que se haya 'geopolitizado' cada aspecto de la implantación. ${ }^{61}$

La rivalidad entre Estados Unidos y China viene reconocida en la Estrategia de Seguridad Nacional de Estados Unidos mencionada anteriormente, la confrontación afecta a todos los campos del poder y de los valores fundamentales. ${ }^{62} \mathrm{El}$ reconocimiento oficial refleja la necesidad de contener, retrasar o impedir el acceso chino al liderazgo mundial, una percepción ya extendida política y socialmente en los Estados Unidos. Las estrategias de comunicación de las Administraciones que alimentan esa percepción recurren a argumentos emocionales de seguridad nacional haciendo hincapié -magnificando incluso- el riesgo de que China utilice la presencia de su tecnología en las redes 5G para vigilar a los ciudadanos y poner en riesgo la propiedad intelectual de sus empresas. Por su parte, las estrategias chinas de comunicación estratégica resaltan el interés estadounidense en obstaculizar el crecimiento económico chino y minusvaloran los riesgos de seguridad. Cualesquiera que puedan ser las razones objetivas de fondo tras el deseo de Huawei de participar en las redes $5 \mathrm{G}$, Estados Unidas y China han entrado en una escalada tecnológica que se añade a otras áreas de competición geopolítica.

Estados Unidos y China se han enfrentado en una guerra comercial que tiene por trasfondo la primacía tecnológica económica y de seguridad. Para mejorar su situación en la competición, Estados Unidos anunció en 2018 una subida de aranceles del 10\% al 25\% a las importaciones chinas para marzo de 2019, a lo que China replicó con subidas similares. Desde entonces, se han mantenido negociaciones, con avances y retrocesos para evitar un enfrentamiento que afectaría a la economía de ambos países y, colateralmente, a la economía global. Las tecnologías figuran entre los objetivos de los aranceles estadounidenses, afectando al $7 \%$ de los productos de alta tecnología y al 55\% de los de tecnología media. Por su parte, los aranceles chinos afectan también a productos tecnológicos estadounidenses, salvo a aquellos como los semiconductores o los productos de aviación que pueden afectar a sus propias cadenas de valor. ${ }^{63} \mathrm{~A}$ los gravámenes con aranceles hay que añadir la posibilidad de que el

\footnotetext{
${ }^{61}$ Triolo, Paul y Allison, Kevin: “The geopolitics of G5”, Eurasia Group White Paper, 15 de noviembre de 2018, en https://www.eurasiagroup.net.

62 "China and Russia challenge American power, influence, and interests, attempting to erode American security and prosperity. They are determined to make economies less free and less fair, to grow their militaries, and to control information and data to repress their societies and expand their influence." National Security Strategy, op. cit., p. 2.,

63“"Our key take-aways on US-china trade war", Natixis Research, China Hot Topics, 10 de septiembre de 2018, en https://www.research.natixis.com.
} 
Departamento de Comercio incluya a compañías chinas en su lista negra de compañías con las que tienen prohibido su relación las compañías estadounidenses (entity list). ${ }^{64}$

El desarrollo de las infraestructuras digitales figura entre los objetivos estratégicos de China, tanto para las que se despliegan en el interior como las que se despliegan en países terceros, especialmente si éstos tienen que ver con la Ruta de la Seda. Estados Unidos alega que las compañías chinas que compiten por desarrollar esas infraestructuras, Huawei y ZTE, no son fiables porque su subordinación a los intereses y políticas del Gobierno chino les puede llevar a implantar 'puertas traseras' en las redes que faciliten ciberataques o poner en riesgo la seguridad nacional. ${ }^{65}$ Estados Unidos ha pedido a sus aliados que excluyan a esas compañías de sus infraestructuras críticas, una exigencia que se ha justificado por razones de seguridad pero que esconde razones de naturaleza geopolítica como la de desplazar a China del mercado global $5 \mathrm{G}$ en beneficio de las empresas estadounidenses.

Las acusaciones contra Huawei y ZTE no son nuevas. Ya en 2012, el Comité de Inteligencia del Congreso llegó a la conclusión de que constituían una amenaza para la seguridad nacional debido a sus intentos reiterados de hacerse con propiedad industrial y a sus conexiones con los servicios de inteligencia, por lo que no se debería permitir su presencia en infraestructuras digitales estadounidenses. ${ }^{66}$ En 2016, Estados Unidos sancionó a ZTE por mantener sus relaciones económicas con Irán y Corea del Norte a pesar del régimen internacional de sanciones en vigor y en 2018 amenazó con prohibir su acceso a microprocesadores fabricados en Estados Unidos, una prohibición que, a diferencia de la impuesta a la compañía Fujian Jinhua Integrated Circuits el mismo año, no se fundaba en el intentos de espionaje industrial a proveedores estadounidenses como Micron, ni en los cuantiosos subsidios recibidos del Gobierno chino por esta compañía para crear su propia fábrica de microprocesadores. En diciembre de 2018, Canadá detuvo a la vicepresidenta de Huawei, Meng Wanzhou, a petición de los Estados Unidos bajo la acusación de violar las sanciones a Irán, la misma razón por la que impuso sanciones a ZTE en abril del mismo año. En 2019, Estados Unidos prohibió a las compañías tecnológicas estadounidenses vender productos y servicios a Huawei. Posteriormente, y en función de la evolución de las negociaciones comerciales con China, la Administración Trump ha ido aplazando o reabriendo su aplicación.

Los argumentos suministrados por las partes son poco contundentes para resolver una disputa emocional y aportan argumentos objetivos junto a otros de difícil objetivación, sembrando la confusión entre capacidades e intenciones. Las compañías chinas alegan que no se ha podido constatar la existencia de puertas traseras ni virus maliciosos en sus productos tecnológicos y han puesto en marcha una campaña internacional de imagen acompañada por la apertura de centros donde se dan facilidades para verificar esas afirmaciones. Las autoridades estadounidenses alegan que la falta de detección de vulnerabilidades en las tecnologías de Huawei no implica que no existan y que la neutralidad de funcionamiento que alega Huawei puede concluir en cuanto el Gobierno chino le pida su colaboración, tal y como obliga la Ley de Inteligencia Nacional china de 2017. Huawei se ha defendido de las acusaciones, emitiendo informes propios y difundiéndolos a través de los centros de trasparencia abiertos en Europa en el que reconoce la posibilidad de que existan riesgos, pero no que no puedan corregirse

\footnotetext{
${ }^{64}$ La última compañía china amenazada de inclusión ha sido Hilkvision, especializada, en vigilancia y reconocimiento facial bajo la acusación de contribuir a la represión de los derechos humanos. Politi, James: "US eyes Hilkvision for export blacklist", Financial Times, 24 de mayo de 2019.

${ }^{65}$ Medin, Milo y Louie, Gilman: “The 5G Ecosystem: Risks \& Opportunities for DoD”, Defense Innovation Board, 3 de abril de 2019, en https://media.defense.gov.

${ }^{66}$ Schmidt, Michael S.: "US. Panel Cites Risks in Chinese Equipment, The New York Times, 8 de octubre de 2012.
} 
aplicando las medidas adecuadas. ${ }^{67}$ Una opinión que parecen compartir las autoridades británicas y alemanas de ciberseguridad que no han recomendado a sus gobiernos secundar la prohibición estadounidense por razones de seguridad nacional.

La disputa entre China y Estados Unidos coge en medio a países, multinacionales y sectores académicos o exportadores que se ven afectadas por las prohibiciones de exportar o de negociar libremente, cuando no se ven obligadas a tomar partido por uno u otro bando, lo que no resulta fácil cuando los intereses económicos están en juego. La UE no ignora los riesgos de seguridad que implica la tecnología china en el control de las infraestructuras 5G, pero no se ha alineado con el bloqueo de Estados Unidos. Esta tendencia a la equidistancia no puede considerarse como una vocación o capacidad de autonomía estratégica, tal y como la practica la Federación Rusa, sino como una resistencia -al menos temporal- a tomar partido siguiendo los dictados de Washington, una resistencia alentada por los desplantes y coacciones que aquejan a las relaciones transatlánticas durante la Administración Trump. Antes de tomar una decisión, la Comisión Europea ha solicitado a los Estados miembros que elaboren un análisis de riesgos y medidas sobre las redes $5 \mathrm{G}$ para contrastarlo con los que van a elaborar la Comisión y la Agencia Europea de Ciberseguridad (ENISA) y presentar una evaluación antes de octubre de 2019. Una vez que se disponga de esa evaluación y de un mecanismo de certificación común, los Estados miembros podrán adoptar las medidas que consideren oportunas para garantizar la seguridad de las redes $5 \mathrm{G}$ dentro del mercado único. ${ }^{68}$

Los aliados han respondido de forma desigual a la demanda de Estados Unidos. Algunos como Australia o Japón accedieron de forma inmediata, pero otros como Canadá, Nueva Zelanda, el Reino Unido, Israel o Alemania han postergado su decisión a la espera de sopesar las razones a favor y en contra de hacerlo. Además de los riesgos para la seguridad, existe el riesgo de que el rechazo a la tecnología china retrase la entrada en funcionamiento de la red 5G o lo encarezca para operadores y usuarios. En marzo de 2019 China dejó de comprar a Canadá semillas de canola y productos cárnicos y arrestó a dos ciudadanos canadienses acusados de espionaje en protesta por la detención de la vicepresidenta Wanzhou de Huawei. ${ }^{69}$ La colaboración canadiense con la petición de extradición estadounidense ha reducido las importaciones mutuas y creado divergencias internas entre empresarios y autoridades. La confrontación también daña colateralmente a la comunidad académica, porque universidades y centros tecnológicos americanos, británicos o australianos han secundado las medidas sancionadoras de Estados Unidos y cortado o reducido su colaboración con las empresas tecnológicas chinas, mientras que otros temen que tengan que acabar optando entre perder las inversiones chinas o ganarse sanciones estadounidenses. ${ }^{70}$

\section{Conclusiones}

La gobernanza multilateral se ve impotente para liderar la transición desde el orden liberal de posguerra hasta el futuro orden global. Su debilidad es a la vez, efecto y causa del distanciamiento de las grandes potencias a las que más benefició. Estados Unidos y China actúan al margen o contra el viejo orden para adaptarse a nuevos retos como la digitalización que no estaba en la agenda de la gobernanza tradicional.

\footnotetext{
${ }^{67}$ Huawei Cyber Security Evaluation Centre, “Annual Report 2019” para la Secretaría del Gabinete del Reino Unido, marzo de 2019, en https://www.gov.uk/government/publications.

68 "La Comisión Europea recomienda un enfoque común en la UE sobre la seguridad de las redes 5G", Comisión Europea, comunicado de prensa, 26 de marzo de 2019, en ", https://europa.eu/rapid/press-release_IP-19- 
La economía digital plantea nuevos retos y oportunidades, pero no iguales para todos, porque beneficia a los más preparados para su adaptación y perjudica a los países y empresas más retrasadas. Las nuevas tecnologías habilitadoras de la digitalización permiten a quienes acceden a ellas desplazar a los que llegan más tarde de las posiciones que ocupaban en la jerarquía internacional. Como resultado, los países y empresas que toman conciencia de la naturaleza disruptiva de las nuevas tecnologías y adoptan estrategias, políticas y medidas para su innovación, desarrollo y explotación.

En ausencia de una gobernanza global efectiva, las iniciativas unilaterales generan un desorden que afecta a los instrumentos, normas e instituciones que controlan la economía global. Grandes potencias como Estados Unidos o China adoptan modelos de confrontación agresivos y 'geopolitizan' todas las dimensiones del poder global, incluidas las tecnológicas, mientras que potencias como la Unión Europea adoptan un modelo más defensivo que trata de preservar el mayor margen de autonomía posible a la espera de una mayor gobernanza multilateral.

La confrontación se agrava por la aceleración del cambio tecnológico, ya que la tecnología multiplica exponencialmente la capacidad de progresar -o descender-, por la escalera de la jerarquía internacional. Con ello aumenta la inseguridad de los países y empresas sobre su prosperidad y estabilidad, por lo que la disrupción tecnológica se convierte en un asunto de seguridad nacional, además de uno de orden global.

Resulta difícil conocer el impacto de las medidas aplicadas, incluso para quienes se deciden a adoptarlas, con lo que aumenta el riesgo de que se produzcan consecuencias no deseadas. A su vez, las contramedidas adoptadas alimentan una escalada de confrontación en la que se ven implicados terceros países o empresas que deben tomar partido por uno u otro bando, como ocurrió durante la Guerra Fría. La formación de alianzas en la nueva guerra fría tecnológica no es fácil porque la disrupción afecta de forma desigual a los actores nacionales, empresariales, industriales o académicos, entre otros, como muestra el desarrollo del caso de Huawei y las redes de $5 \mathrm{G}$.

Las medidas unilaterales afectan a las relaciones internacionales, tal y como se entendían hasta ahora, y obligan a potenciar el estudio de las cuestiones tecnológicas y científicas dentro la disciplina de Relaciones Internacionales, para evitar su retraso sobre otras disciplinas como la Ciencia Política, la Economía o los Estudios de Seguridad que ya se han dedicado a la investigación y teorización de las tecnologías disruptivas y el orden global. Seguro que a Paloma García Picazo le hubiera gustado dedicarse a ello. 


\section{Bibliografía}

"A Global Strategy for the European Union's Foreign and Security Policy. Shared Vision, Common Action: A Stronger Europe", Consejo Europeo (2018): en https://eeas.europa.eu.

"A Next Generation Artificial Intelligence Development Plan", Consejo de Estado Chino: traducción, en https://www.newamerica.org.

“Annual Report 2019”, Huawei Cyber Security Evaluation Centre, marzo 2019, en https://www.gov.uk/government/publications.

Arteaga, Félix (2018): "La cuarta revolución industrial (4RI): un enfoque de seguridad nacional", Documento de Trabajo del Real Instituto Elcano, $\mathrm{n}^{\circ}$ 12, en http://realinstitutoelcano.org

"Assessing and Strengthening the Manufacturing and Defense Industrial Base and Supply Chain Resiliency of the United States", Interagency Task Force in Fulfillment of the Executive Order 13806, septiembre de 2018, en https://media.defense.gov.

Banco Mundial (2019): "High-Tech Exports”, en https://data.worldbank.org.

Beck, Ulrich (2006), La sociedad del riesgo global, Madrid, Siglo XXI.

Bine Pascal, Bernardini Sandro de, Horbach Matthias, Leiter Michael E., Schlager Ivan A., Weinbaum Michelle A.:"Expanding the Scope of National Security-Focused Foreign Investment Reviews in Europe”, julio 2018, Skadden, Arps, Slate, Meagher \& Flom LLP, en https://www.skadden.com.

Buckley, Chris y Porter, Catherine: "China Accuses Two Canadians of Spying, Widening a Political Rift", The New York Times, 4 de marzo 2019.

Bueguer, Christian (2018): "Security as practice", en Myriam Dunn Cavelty (ed.), Routledge Handbook of Security Studies, Taylor and Francis, p. 131.

"Business cannot ignore the US-China Cold War", Financial Times, 10 de junio de 2019.

Calhoun, Paul : "DARPA Emerging Technologies", Strategic Studies Quarterly,( otoño 2016) pp. 91-113.

Cavelty, Myriam Dunn; Fischer, Sophie-Charlotte y Balzaq, Thierry (2018): "Killer Robots and Preventive Arms Control" en Myriam Dunn Cavelty (ed.), Routledge Handbook of Security Studies, Taylor and Francis, pp. 457-459.

Conn, Ariel : "The United Nations and the Future of Warfare", Bulletin of the Atomic Scientist, 9 de mayo 2019, en https://thebulletin.org.

“Control de las inversiones extranjeras directas en la Unión”, Reglamento (UE) 2019/452 de 19 de marzo de 2019, Parlamento Europeo y Consejo, en https://eur-lex.europa.eu.

Cremer, Jacques; De Montjoye, Yves-Alexandre y Heike Schweitzer, Heike (2019): "Competition policy for the digital age", en http://ec.europa.eu/competition.

Czerwonka, Michal (2019): “How Chinese Spies Got the N.S.A.'s Hacking Tools, and Used Them for Attacks", The New York Times, 6 de mayo.

Danilin, Ivan V. (2018): “Emerging Technologies And Their Impact On International Relations and Global Security", Hoover Institution Fall Series, $n^{\circ} 118$, en https://www.hoover.org/publications.

"Datos y cifras de la OMPI sobre PI", edición en español, Organización Mundial de la Propiedad Industrial (2018): pp. 9-11. 
"European Council EU-China. A strategic outlook ", Comisión Europea y Alta Representante: JOIN COM (2019) 5 final, 12 de marzo, en https://ec.europa.eu/commission.

"The age of artificial intelligence", European Political Strategy Center, EPSC Strategic Notes, (2019) $n^{\circ} 29$, p. 4.

Eagler, John y Pritzker, Penny (2018): "The Work Ahead: Machines, Skills, and U.S. Leadership in the Twenty-First Century", Council on Foreign Relations Independent Task Force Report, $n^{\circ} 76$, abril, pp. 16-17, en https://cfr.org.

Edgerton, Davies: "The Contradictions of Techno-Nationalism and Techno-Globalism. A Historical Perspective”, New Global Studies, vol. 1, no 1 (enero 2017), pp. 34-39.

"FY 2020 Administration Research and Development Budget Priorities", Memorandum M18-22 de 31 de julio 2018, The White House, en https://whitehouse.gov.

Farrel, Henry y Newman, Abraham L.: "Weaponized Interdependence: How Global Economic Networks Shape State Coercion”, International Security, vol. 44, n 1, (verano 2019) pp. 4279.

Feng, Emily: "How China acquired mastery of vital microchip technology, Financial Times, 29 de enero 2019.

Freier, Nathan: "The Emerging Anti-Access/Area-Denial Challenge”, CSIS Critical Questions, 17 de mayo 2012, en https://www.csis.org

Gobierno de Francia (2019): "La croissance et la transformation des entreprises", Ley 486 de 22 de mayo, en https://www.legifrance.gouv.fr.

Gilli, Andrea y Gilli, Mauro (2019): "Military-Technological Superiority and the Limits of Imitation, Reverse Engineering, and Cyber Espionage", International Security, vol. 43, n 3, p. 144.

"Inteligencia artificial para Europa", Comisión Europea, COM (2018) 237 final de 25 de abril, en https://ec.europa.eu

Jaske, Alex: "Picking Flowers, Making Honey", Australian Strategic Policy Institute, 30 de octubre, en https://aspi.org.au.

Jost Wübbeke et al.: "Made in China 2025. The making of a high-tech superpower and consequences for industrial countries", Mercator Institute for China Studies, MERICS Papers on China, $\mathrm{n}^{\circ}$ 2, December 2016, en https://www.merics.org.

Kennedy, Scott : “The Fact Tec Dragon. Benchmarking China's Innovation Drive”, CSIS China Innovation Policy Series, August 2017, p.1, en https://www.csis.org.

"La Comisión Europea recomienda un enfoque común en la UE sobre la seguridad de las redes 5G", comunicado de prensa, Comisión Europea, 26 de marzo de 2019, en https://europa.eu/rapid/press-release_IP-19-1832_es.htm

Lee, Thimothy B.: "Here's everything we know about PRISM to date", The Washington Post, 12 de junio 2013.

Leonard, Mark: "Conectivity Wars", European Council of Foreign Relations, enero 2016, pp. 13-21, en https://ecfr.eu/.

Leonard, Mark et al.: "Redefining Europe's economic sovereignty, Bruegel Policy Contribution, $\mathrm{n}^{\circ}$ 9, (2019), en http://bruegel.org 
Lind, Jennifer: "The Rise of China and the Future of Transatlantic Relationship", Chatham House Briefing, 29 de julio 2019, p. 5.

Lucas, Louise; Liu, Nicole y Sender, Henny: "MIT breaks with Huawei and ZTE as it heightens China scrutiny", Financial Times, 5 de abril 2019.

Mayer, Maximilian: "The Global Politics of Science and Technology: An Introduction", en Mayer Maximilian; Carpes, Mariana y Knoblich, Ruth (eds.) (2014), The Global Politics of Science and Technology, vol. 1, Mayer, Springer, pp. 2-3.

McDonald, Sean y Mina, An Xian: “The War-Torn Web”, Foreign Policy, 19 de diciembre 2018, en https://foreigpolicy.com.

McKinsey: "Disruptive technologies: Advances that will transform life, business, and the global economy”, McKinsey Global Institute, mayo 2013, pp. 4-5.

Mearsheimer, John J.:"Bound to Fail: The Rise and Fall of the Liberal International Order", International Security, vol. 43, nº 4, (primavera 2019), pp. 7-50.

Medin, Milo y Louie, Gilman: “The 5G Ecosystem: Risks \& Opportunities for DoD”, Defense Innovation Board, 3 de abril 2019, en https://media.defense.gov.

Morrison, Wayne M.: "China-U.S. Trade Issues", Congressional Research Service, 6 de julio 2018 , p. 39.

"National Defence Strategy", sumario no clasificado, Departamento de Defensa de los Estados Unidos (2018), p.3, en https://dod.defense.gov

"National Security Strategy of the United States of America, 18 de diciembre 2017, The White House, en https://whitehouse.gov.

Ortega, Andrés: "Europa se quita el velo chino", Blog del Real Instituto Elcano (abril 2019), en https://realinstitutoelcano.org.

"Our key take-aways on US-china trade war", Natixis Research, China Hot Topics, 10 de septiembre de 2018, en https://www.research.natixis.com

Plender, John: "The threat of a US-China currency war", Financial Times, 6 de agosto 2019.

Politi, James: "US eyes Hilkvision for export blacklist", Financial Times, 24 de mayo 2019.

Potter, Alex: "United Kingdom", en Goldman Calvin (ed.) (2018): The Foreign Investment Regulation Review, sexta edición, Londres, Law Business Research Ltd, pp. 195-226.

"Rethinking Strategic Autonomy in the Digital Age", European Political Strategic Centre, EPSC Strategic Notes, 2019, n 30, p. 1, en https://ec.europa.eu/epsc.

Schmidt, Michael S.: "US. Panel Cites Risks in Chinese Equipment, The New York Times, 8 de octubre 2012.

Schneider-Petsinger, Marianne: "US-EU Trade Relations in the Trump Era Which Way Forward?", Chatham House US and the Americas Programme, 20 March 2019 en http://chathamhouse.org

Schwab, Klaus (2017): The Fourth Industrial Revolution, Nueva York, Crown Business.

Stanton, Charlotte et al.: "What the Machine Learning Value Chain Means for Geopolitics", Carnegie Endowement, August 2019, en https://carnegieendowment.org.

“The Defence Innovation Initiative”, Memorandum de 15 de noviembre 2014, Department of Defence, en https://archive.defense.gov. 
"The Impact of the ICT Revolution on International Relations", en Strategic Survey 2018: The Annual Assessment of Geopolitics, Londres, IISS, p. 34.

"The US and the Iran Nuclear Deal: rejoining is wiser than destroying", Joint Call of European Think-Tanks, Elcano Royal Institute, 8 de junio 2019, en http://realinstitutoelcano.org.

Triolo, Paul y Allison, Kevin: "The geopolitics of G5”, Eurasia Group White Paper, 15 de noviembre 2018, en https://www.eurasiagroup.net.

Uren, Tom; Hogeveen, Bart y Hangus, Ferson: "Defining offensive cyber capabilities", Australian Strategic Policy Institute, 4 de julio 2018, en https://www.aspi.org.au.

Weiss, Charles: "How Do Science and Technology affect International Affairs", Minerva: A Review of Science, Learning and Policy, vol. 53, n ${ }^{\circ}$ 4, (2015) pp. 411-430.

Yang, Yuan y Murgia, Madhumita: "Microsoft under fire over AI work with chines military university", Financial Times, 11 de abril 2019.

Yoplensky, Rochelle y Murgia, Madhumita: "EU antitrust crackdown on Big Tech Urged", Financial Times, 5 de abril 2019.

Yuan, Li: “As Huawei Loses Google, the U.S.-China Tech Cold War Gets Its Iron Curtain”, The New York Times, 20 de mayo 2019. 